glutamyl glycine ethyl ester, m.p. $107-109^{\circ}$, which was saponified to carbobenzoxy $\gamma$-L-glutamyl glycine, m.p. $159-161^{\circ}$ (found : C, 53.5; H, 5.3; N, 8.3; $\mathrm{C}_{15} \mathrm{H}_{18} \mathrm{O}_{7} \mathrm{~N}_{2}$ requires: $\mathrm{C}, 53 \cdot 24 ; \mathrm{H}, 5 \cdot 37 ; \mathrm{N}, 8 \cdot 28$ per cent). Hydrogenation gave $\gamma$-L-glutamyl glycine, m.p. 193-194 (found: C, 41.5; H, 5.9; N, 13.7; $\mathrm{C}_{7} \mathrm{H}_{12} \mathrm{O}_{5} \mathrm{~N}_{2}$ requires : $\mathrm{C}, 41 \cdot 17, \mathrm{H}, 5.93 ; \mathrm{N}, 13 \cdot 72$ per cent. $[\alpha]_{D} D^{14}+11 \cdot 1^{\circ} \pm 0 \cdot 8^{\circ}$ in water, $\left.c, 2 \cdot 5\right)$.

Partition chromatography on paper (using phenol saturated with water as the mobile phase) confirmed the purity of the $\gamma$-L-glutamyl glycine, in so far as only one component was revealed by ninhydrin. With the latter reagent at $p \mathrm{H} 2.5$ and $100^{\circ}, 1 \cdot 0$ mole of carbon dioxide was evolved, confirming the presence of a free $\alpha$-amino-acid group.

These investigations are being continued and extended to the aspartic acid analogues. We are grateful to the Government Grants Committee of the Royal Society for financial assistance.

W. J. Le QUESNE

G. T. Young

Dyson Perrins Laboratory,

Oxford.

Oct. 23.

${ }^{1}$ Bergmann, M., and Zervas, L., Ber., 65, 1192 (1932).

${ }^{2}$ Bergmann, M., Zervas, L., and Fruton, J. S., J. Biol. Chem., 115, 606 (1936).

3 Melville, J., Biochem. J., 29, 179 (1935).

- Bergmann, M., Zervas, L., Salzmann, L., and Schleich, H., Z. physiol. Chem., 224, 17 (1934).

'Aberhalden, E., and Nienburg, H., Z. physiol. Chem., 219, 155 (1933).

Bergmann, M., and Zervas, L., ibid.,.221, 51 (1933). Nienburg, H., Ber., 68, 2232 (1935).

'Hegedius, B., Helv. Chim. Acta, 31, 739 (1948).

\section{Comparative Study of Sucrose Inversion and Synthesis by Carrot and Radish Root Slices}

IN previous papers ${ }^{1,2}$, it has been established that when radish or carrot root slices were suspended in sucrose solution, the latter was always inverted in the medium before its absorption by the tissue slices, and the inversion was always faster than the uptake, hence the accumulation of invert sugar in the culture medium. Gawadi ${ }^{3}$ and Burström ${ }^{4}$ showed that sucrose inversion takes place by means of invertase centres attached to the outer cytoplasmic surfaces of cells of the slices. Saïd ${ }^{1}$ found that when slices of

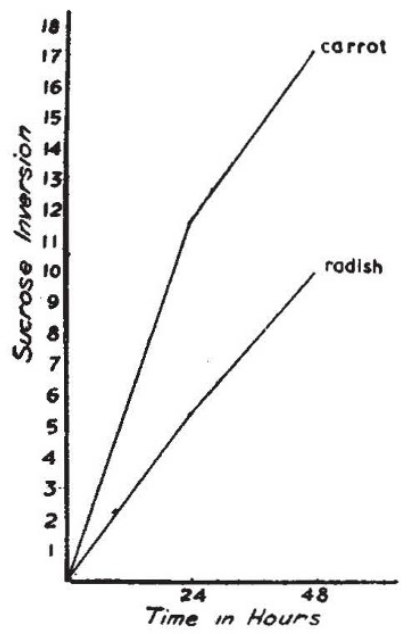

Total sucrose inversion in the culture media of equal numbers of carrot and radish root slices having the same diameter varied thicknesses were suspended in sucrose solution, the rate of inversion in the medium was directly proportional to the number of exposed surfaces per mass of tissue slices.

We carried out several experiments in which l-mm. thick slices of carrot and radish roots, having the same diameter, were suspended in sucrose solutions. Comparing the rates of sucrose inversion by equivalent numbers of slices of both roots, it was found that the rate of sucrose inversion by carrot slices was always much faster than that by radish slices (see accompanying graph). This observation leads to the conclusion that the number of active invertase centres on the outer cytoplasmic surfaces of radish is less than on equivalent surfaces of carrot.

Analysis of the sugar-fed slices from these two plants showed further that carrot slices always build up sucrose from any absorbed sugar whereas radish slices form but little sucrose from the absorbed sugars, the accumulation being always in the form of hexoses.

Botany Department,

H. SAÏD

H. FAWZY

Faculty of Science,

Fouad I University, Cairo, Egypt. Feb. 21 .

${ }^{1}$ Said, H., Bull. Fac. Sci., Cairo, 24, 31 (1941).

${ }^{2}$ Saĩd, H., Bull. Fac. Sci., Cairo, 25, 117 (1945).

${ }^{8}$ Gawadi, A. G. H., Ph.D. thesis (University of Cambridge, 1935).

' Burström, H., Ann. Agric. Coll. Sweden, 9, 264 (1941).

\section{Coagulation of Oxalated Plasma Without Calcium Following Neutralization of Heparin by Toluidine Blue}

UNDER physiological conditions, calcium is necess. ary for the coagulation of blood, because with thromboplastin it brings about the change of prothrombin into thrombin. But the true role of calcium ions has not hitherto been understood. On the other hand, this change is inhibited by heparin in connexion with a plasmatic co-factor, which was determined as an acid-soluble albumin ${ }^{1}$. The anticoagulating influence of heparin is specifically inhibited by toluidine blue ${ }^{2}$. We have used this property of toluidine blue to examine the function of the heparin which in normal circumstances is present in the blood. The influence of the physiological amount of heparin on blood coagulation is still under discussion. The complex heparin-plasma co-factor inhibits the action of thromboplastin on the change of prothrombin to thrombin ${ }^{3}$. We are putting forward the working hypothesis that the interaction between the heparin complex and thromboplastin of plasma is the cause of the fluidity of blood in the vessels, and that calcium in some way either with a tissue extract or otherwise neutralizes the heparin complex. If this assumption is correct, the toluidine blue will coagulate the decalcified plasma.

Oxalated pig plasma was used, and the point of coagulation determined in a water bath at $37^{\circ} \mathrm{C}$. Toluidine blue was added in the isotonic Michaelis veronal buffer with sodium chloride in various con. centrations. Fig. 1 shows the rate of coagulation of plasma induced by toluidine blue without recalcification. Further, we found that the clotting time under the influence of toluidine blue (curve $A$, Fig. 2) depends on the dilution of the plasma in a similar 